\title{
Hemiprosthesis for Femoral Neck Fractures in the Elderly: A Retrospective Study of 319 Patients
}

\author{
Axel Prokop, ${ }^{1, *}$ and Marc Chmielnicki ${ }^{1}$ \\ ${ }^{1}$ Clinic for Trauma Surgery, Hospital Sindelfingen, Sindelfingen, Germany \\ "Corresponding author: Axel Prokop, Clinic for Trauma Surgery, Hospital Sindelfingen, Sindelfingen, Germany. Tel: +49-70319812422, Fax: +49-70319812842, E-mail: \\ verletzt@klinikverbund-suedwest.de
}

Received 2015 September 26; Revised 2016 March 30; Accepted 2016 May 07.

\begin{abstract}
Background: In geriatric patients with Pauwels types II and III femoral neck fractures, hemiprosthesis is the therapy of choice. Objectives: This study was conducted to analyze the results after cemented hemiprosthesis placement, the first year after surgery. Patients and Methods: This retrospective study was conducted on 319 patients over 70 years with displaced femoral neck fractures treated surgically at our hospital from 2007 to 2012. All medical information was available including retrospective posthospital discharge records as well as inpatient course and one-year mortality.

Results: From a total of 319 patients, $78 \%(n=249)$ were female and $22 \%(n=70)$ were male, with the mean age of 83.6 years. Seventeen percent of the patients suffered from heart failure, $23 \%$ from diabetes, and $19 \%$ from renal insufficiency. Time to surgery averaged one day postinjury. Average operative time skin-to-skin was 50 minutes. Seventy-three percent of the patients could mobilize independently on discharge. Of the remaining patients, $2 / 3$ had already lost independent mobilization prior to the fracture. Hospital mortality averaged 5\% (national average in Germany: 8\%), and 30-day and 90-day mortality rates were 5\% and 15\%, respectively. Within one year, $22 \%$ of the patients died (national average: $27 \%$ ). Also, 14 patients were re-admitted, for contralateral prosthetic implantation $(n=7)$ or revision after the periprosthetic fracture $(n=5)$. Fifty-three percent of the patients were admitted to hospital during the year for other diseases (national average: $54 \%$ ).

Conclusions: Hemiprosthesis placement for displaced femoral neck fractures is a common and safe procedure. Despite recent decreases in hospital mortality, the risk of death remains more than twice as high within one year than that for uninjured patients of the same age.
\end{abstract}

Keywords: Geriatric, Femur Fracture, Hemiprosthesis, Trauma in Elderly

\section{Background}

Femoral neck fractures are typical and frequent injuries in the elderly. Because of osteoporosis-reduced bone density and increasing varus deformity, the femoral neck is predisposed to fractures, even after minimal trauma $(1,2)$. The incidence increases dramatically with age. In Germany, this injury occurs in $90 / 100,000$ population (35 ). For those over 65 , the incidence is $966 / 100,000$ population, with figures varying based on the region of residence. For example, in Baden-Württemberg, the incidence, with 507/100,000, is significantly less than in Hamburg, with $1404 / 100,000$ population. It is still under discussion whether this has to do with sun exposure and vitamin D production (6). A five-fold increase in incidence is expected by 2050 (4).

The cost of medical treatment amounts to approximately $20,000 €$ per fracture or 5.4 million $€$ for all cases in Germany (6). As was the case twenty years ago, these fractures lead to a significant reduction in life expectancy and often result in dramatic deterioration in social and health functioning of affected patients (7). Many patients lose their independence and require external assistance, or even need full-time nursing care (8).

The fractures are classified according to Stockle et al. and Garden. Pauwels evaluates the slope of the fracture line. This study involves Pauwels II and III and Garden 3 and 4 fractures only. According to the AO Classification, this involves fractures 31B2 and 31B3 $(2,9,10)$.

Pauwels types II and III as well as Garden types III and IV fractures are thus absolute indications for surgery. While these fractures can be treated with head-sparing procedures in patients under 65, the consensus is to implant an endoprosthesis in patients over 70, to avoid later femoral head necrosis (5).

Hemiprosthesis, without acetabular replacement (bipolar prosthesis) has less blood loss and shorter operative time than total arthroplasty, and is preferred in elderly 
patients over 75 - 80 years (Figures 1 and 2). Although the ten year long-term results are worse than with total hip arthroplasty, they are preferred in older and diseased patients because loosening or other acetabular changes are no longer experienced. In addition, the dislocation rate is lower in bipolar prostheses, so that patients can be mobilized more quickly and without limitations (5). These numbers have been confirmed also in the large Swedish, Finnish, and Norwegian prosthesis registries (11-14).

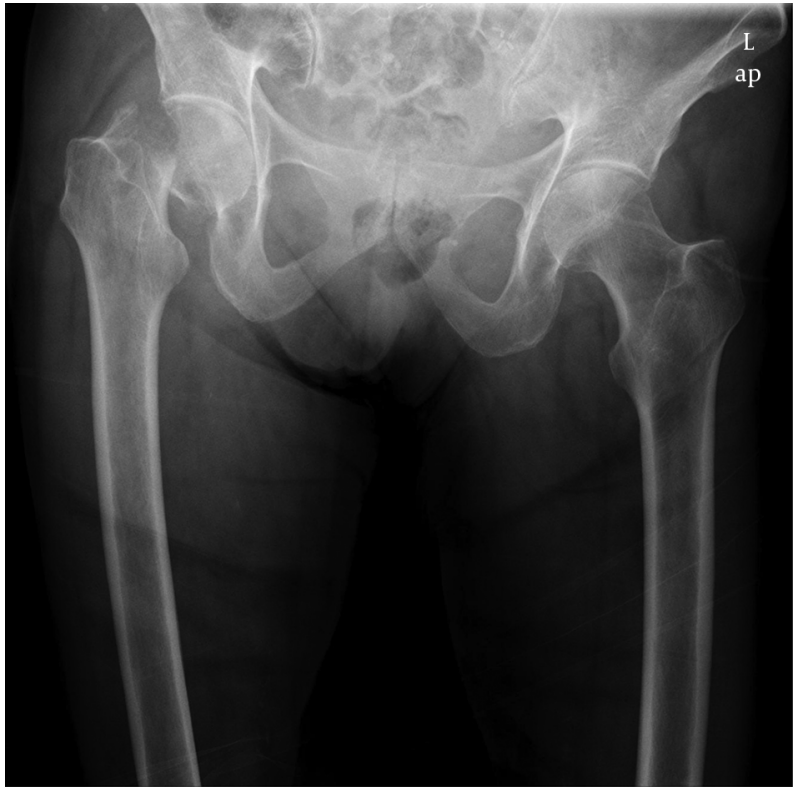

Figure 1. Female, 77 Years Old Preoperative, X-Ray Hip, Pauwels III-Fracture

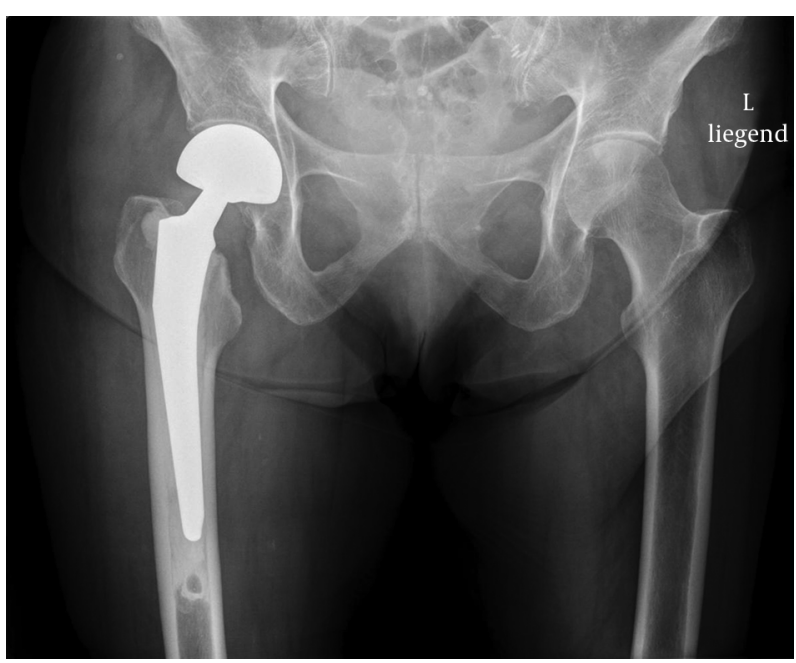

Figure 2. Postoperative X-Ray With Hemiprosthesis

\section{Objectives}

The aim of the present study was to analyze one-year results after cemented hemiprosthesis placement, the first year after surgery.

\section{Patients and Methods}

This study was a retrospective study. In cooperation with the largest German health insurance company, the Allgemeine Ortskranken Kasse (AOK), data were collected from all patients with displaced femoral neck fractures treated operatively with prostheses in our clinic from 2007 to 2012 .

All patients over 70 years with Pauwels type II and III fractures receiving a hemiprosthesis were included. Only patients under 70 years of age were excluded.

The AOK provided all medical records of all patients diagnosed with femoral neck fracture for one year following injury, enabling 100\% follow-up. The data were collected entirely by the AOK for our clinic. The AOK is responsible for compliance to privacy regulations and the consent to processing and publication of patient data.

In addition to mortality, registered information included all hospital re-admissions for complications or follow-up surgeries to the femoral neck fracture within the first year.

Due to the increased age of the patients with already limited life expectancies, follow-up longer than one year was not possible.

Three hundred and nineteen patients underwent surgery (our own operative video of the bipolar prosthesis (15)). All 319 patients were followed either until death or for one year postsurgery.

This was according to the information from the health insurance company, with $100 \%$ complete follow-up for one year regarding mortality and hospital readmission.

With this number of included patients, we were in the 97th percentile of all German hospitals.

In this study, Microsoft Excel and SPSS were used to calculate mean and standard deviation values. All patients' data were entered into Excel tables, and duplicates were eliminated through sorting by name and birth date.

The text was written with Microsoft Word. The ethics commission of the southwest hospital association approved the retrospective study.

\section{Results}

From a total of 319 patients, $78 \%(n=249)$ were female and $22 \%(\mathrm{n}=70)$ males, with the mean age of $83.6 \pm 8.7$ (70 - 98) years. Twenty percent $(n=64)$ of the patients had 
the patient complication and comorbidity level (PCCLs) of 3 and $45 \%(n=144)$ of 4 points; $17 \%(n=54)$ suffered from heart failure, 23\% $(n=70)$ from diabetes, and 19\% $(n=61)$ from renal insufficiency (Table 1 ).

Results of the current study showed that the average operative time skin-to-skin was $50 \pm 6$ minutes. Hematoma or seroma occurred in $45 \%(n=144)$ of the patients. Also, $8 \%(n=25)$ of the patients had pneumonia, pulmonary embolus in 1.5\% ( $n=5)$, and infection in 1.5\% ( $n=$ $5)$. The prosthesis dislocated in $3 \%(n=10)$ of the cases and $4 \%(n=13)$ of the patients required ventilation for over 24 hours. Ninety-six percent of the patients were transferred to a regular care floor from the recovery room.

The average duration of admission was 14 days in 2007, and decreased to 12 days in 2012 due to optimization through introduction of discharge management.

At discharge, $73 \%(n=232)$ of the patients could mobilize independently. Specifically, this means that patients, with or without a walker, were able to walk unassisted on the hospital floor as well as up and down at least one flight of stairs. Of the remaining patients, $2 / 3$ had no longer been mobilizing independently prior to the fracture, either because they were bedridden in a nursing facility, or because they were confined to a wheelchair due to other causes or comorbidities.

Hospital mortality was 5\% (16 patients) (national German average $8.1 \%$ ), and at 30 and 90 days was $5 \%$ and $15 \%$, respectively. Twenty-two percent of the patients $(n=70)$ died within one year (national German average 27\%), with a natural death probability of $7.1 \%$ at 83.5 years (16). The most frequent cause of death was cardiovascular disorder. After surgery, 14 patients were re-admitted for contralateral prosthesis placement (7 patients) or for periprosthetic fracture (7 patients). Prosthetic displacements were seen only during the first ten postoperative days $(n=10)$, but not thereafter during the course of the year (Table 2 ).

Results showed that $54 \%$ of the patients were readmitted to hospital within one year for another condition (national German average 54\%).

\section{Discussion}

Hemiprosthesis is a good treatment for femoral neck fractures in geriatric patients. The procedure is performed quickly and safely.

The bipolar prosthesis has very good immediate results for patients aged $70-75$ years $(16,17)$. The patients can be immediately mobilized postoperatively with full weight-bearing. Because of the size and mobility of the head, dislocations are rare. Because there is no need for cup preparation, operative times are markedly shorter than in total hip arthroplasty.
Follow-up for patients with hip prostheses is generally at least 5 to 10 years. In our patient population, the patients were very old and often with a significant comorbidity, so that the primary goal of treatment was rapid mobilization with full weight bearing and return to the pre-injury environment. Because of the limited life expectancy, follow-up was limited to one year postoperatively.

In the literature as well, for this reason there are no long-term follow-up studies of these geriatric patients.

Schleicher et al. reported this in a prospective study. Fifty-four patients with an average age of 80.5 years were treated with a total arthroplasty and 52 patients with an average age of 81 years with a bipolar prosthesis. Operative times, blood loss, units of blood replacement required, and hospital mortality were significantly less in patients receiving bipolar prostheses. At a follow-up examination after 8 years, however, the surviving patients who had undergone total arthroplasty were significantly better functionally than patients receiving the bipolar prosthesis (18). The guidelines of the Association of the Scientific Medical Societies in Germany (AWMF) come to similar results and recommendations (5).

Despite safer operative techniques, there are frequent complications in the very elderly and infirm patients. Muller et al. investigated data from 204 patients after bipolar implants with an average age of 82 years. Postoperatively, 15\% had relevant surgical complications requiring revision (5\% infections, 5\% hematomas, and 3.4\% dislocation). Cardiovascular, renal, and neurologic complications occurred in $43 \%$ of the patients. In a follow-up examination after an average of 28 months, 48 patients had died (19). The optimal delay to operation remains under intense debate. Early operation allows more rapid mobilization from bed. However, emergency staffing on weekends and overnight may prevent this. The most experienced surgeon is not always present. Schliemann et al. investigated these factors in a retrospective study of 360 bipolar prostheses. They compared the outcomes after surgery which was performed by a "senior surgeon" versus that performed by a "junior surgeon." The authors found that the skin-to-skin operative time for experienced surgeons, with 61 minutes, was significantly shorter than that for less-experienced surgeons, with 77 minutes. This difference was highly statistically significant $(\mathrm{P}<0.001)$. Revision surgery was necessary in 25 cases. More complications were observed in the group treated by the junior surgeons, with 9.6\% (versus 6.3\% in those treated by senior surgeons). However, this difference was not statistically significant. Operations performed at night did not result in more complications than those performed during the day (20). For all operations in our clinic, a senior surgeon was present as first assistant to offer experienced help to the younger 
Table 1. Demographic Information of the Patients ${ }^{\mathrm{a}}$

\begin{tabular}{lccc}
\hline Patients & Age, $\mathbf{y}$ & Comorbidity & Diseases \\
\hline $\mathbf{N}=$ 319; & $83.6 \pm 8.7$ & $64(20)$ PCCL 3 & $70(23)$ diabetes \\
$\mathbf{M : F}=\mathbf{7 0 : 2 4 9}$ & $70-98$ & $144(45)$ PCCL 4 & $61(19)$ renal insufficiency \\
& & & 54 (17) heart failure \\
\hline
\end{tabular}

Abbreviations: f, female; $\mathrm{m}$, male; PCCL, patient complication and comorbidity level.

${ }^{a}$ Values are expressed as No. (\%)

Table 2. Results ${ }^{\mathrm{a}}$

\begin{tabular}{|c|c|c|c|}
\hline Patients & Operative Time, min & Complications & Mortality Rate \\
\hline \multirow{5}{*}{$\mathbf{N}=\mathbf{3 1 9}$} & \multirow{5}{*}{$50 \pm 6$} & Hematoma $(n=144)$ & Hospital 16(5) \\
\hline & & Pneumonia $(n=25)$ & 30 days $+0(5)$ \\
\hline & & Infection $(\mathrm{n}=5)$ & 90 days $+31(15)$ \\
\hline & & Displacement $(\mathrm{n}=10)$ & 1-year +22 (22) \\
\hline & & & Total: $69 / 319$ patients within 1 year \\
\hline
\end{tabular}

${ }^{\mathrm{a}}$ Values are expressed as No. (\%).

surgeon and limit operative time as well as complications.

In our clinic, we treat many patients with femoral neck fractures and proximal femur fractures. As a center for trauma of the elderly, this is a focus of our clinic. Thus, with this number of included AOK patients, we were in the 97th percentile of all German hospitals. This explains also the shorter average operative time of 50 minutes. All patients are operated in the first possible daily schedule time slot. Perhaps this is also the reason for slightly lower hospital mortality rate of $5 \%$, compared to the national German average of $8 \%$. We believe that surgery performed at the earliest possible time as well as structured hospital protocols decrease mortality. As long as patients are waiting for surgery, they are confined to the bed because they cannot stand on the fractured leg. Each day's delay to surgery leads to frequent complications such as pressure ulcers, pneumonias, and cardiovascular disorders and therefore deteriorations of health and increased risks. Unfortunately, statistical analysis of mortality according to age, gender, and comorbidity was not performed.

In an investigation of all patients undergoing surgery in our clinic for proximal femur fractures between 2007 and 2012, mortality for patients operated within 24 hours was just $4.1 \%$ and increased to $7.2 \%$ when surgery was performed after 24 - 48 hours. For this reason, we made efforts in the past few years to establish and structure care protocols. Treatment pathways were introduced to delineate the course of patient care from the Emergency and Radiology departments through surgery, discharge man- agement and back to home. Anesthesiologists and geriatricians were consulted for measures to expedite patient surgical-readiness. Supplementary cardiologic consultations could be almost completely foregone.

Smektala et al. (7) published data from a Germanywide survey of the years 2004 - 2006. 129,075 patients with femoral neck fractures were evaluated. Of these, 22,171 patients were operated after 48 hours. In the group operated later than 48 hours postinjury, there were significantly more frequent surgical complications [odds ratio OR 1.1 (95\% CI 1.03 - 1.18)], general complications [OR 1.09 (95\% CI 1.04 - 1.14)], and decubital ulcers [OR 1.27 (95\% CI $1.15-1.40)]$. However, the hospital mortality did not change significantly (5.0\% versus $6.2 \%$ for all patients, $6.2 \%$ versus $6.5 \%$ after statistical matching) compared to patients operated earlier [OR 0.96 (95\% CI $0.89-1.04)$ ]. No distinction was made among operations performed within the first 24 hours (7).

Thus, does it make sense to introduce specialty centers or minimum quantitative limits for treatment of femoral neck fractures. Schrader et al. investigated this question. Using the incidence, they developed a model calculation on the effects of the national coverage with prostheses in fractures and coxarthrosis. Seventeen percent of all German hospitals $(n=216)$ implant a maximum of 20 prostheses annually. This corresponds to 2214 patients and thus $1.4 \%$ of all cases. In $38 \%$ of all German hospitals, a maximum of 50 prostheses are implanted. This corresponds to 11,476 patients, or $7.4 \%$ of all cases (21). This means that 
most patients (81.2\%) are already treated in hospitals performing more than 50 prosthesis implantation surgeries per year.

There are few figures regarding the long-term course of these sick and elderly patients with femoral neck fractures. The thorough data collection of the AOK makes this possible for the first time. Although the AOK data allow no conclusions regarding concomitant illnesses such as dementia, delirium, depression, etc., because this information was not simultaneously collected, conclusions regarding mortality can be made. Thus, the high one-year mortality rate remains alarming.

Within our own patient collective of AOK patients, 22\% of the patients died within one year. Even comparing this with mortality tables of comparably old patients with an average age of 83.5 years, there is a natural mortality rate of $7.1 \%$ (1). Thus, there is an almost three-fold higher risk of dying within the first year after a femoral neck fracture than there is without the injury. The goal of treatment must be the reintegration of geriatric patients to their previous environment, whenever possible.

In 2010, Schneppendahl et al. investigated 530 patients undergoing bipolar hemiprosthesis placement from the years 1989 - 2003, with an average age of 81 years; 145 of 175 surviving patients were followed up after an average 91 months and 355 patients had already died by this time. In $78 \%$ of the cases, the investigated patients had reached their preoperative mobility status, although $68 \%$ of the patients required previously unnecessary assistance devices and 32\% required advanced nursing care. Sixty-eight percent of the patients were, however, able to return to their preoperative surroundings (22).

In our patient group, $73 \%$ were able to mobilize independently, with assisting devices when necessary. Of the 27\% who could not mobilize independently, however, $75 \%$ had been previously non-mobile prior to the injury.

Other authors have reported similarly good results after total hip replacement compared to hemiprosthesis in the elderly. Blomfeld et al. reported such results after a randomized comparison study of 120 patients with mean age of 81 years (70 to 90) and acute displaced intracapsular fractures of the femoral neck. They were randomly assigned for treatment with either bipolar hemiarthroplasty or total hip replacement. Outcome measurements included perioperative data, general and hip-specific complications, hip function, and health-related quality of life. The patients were re-evaluated at four and 12 months. The duration of surgery was longer in the total hip replacement group (102 minutes (70 to 151)) versus 78 minutes ( 43 to 131) $(\mathrm{P}<0.001)$, and the intra-operative blood loss was increased $460 \mathrm{~mL}$ (100 to 1100) versus $320 \mathrm{~mL}$ (50 to 850$)(\mathrm{P}<0.001)$, but rates of complications or mortality did not differ between the groups. There were no dislocations in either group. Hip function measured by the Harris hip score was significantly better in the total hip replacement group at 4 and 12 months after surgery $(\mathrm{P}=0.011$ and $\mathrm{P}<0.001$, respectively) (23). Other authors have reported similar results $(24,25)$.

With our somewhat older patients, we followed the strategy of minimizing operative risks and therefore, selected the shorter operative times and minimized blood loss, with a displacement rate of $0 \%$ in our own patient group.

One weakness of this study was the lack of comparison with a patient group undergoing total hip replacement, as well as the lack of functional follow-up and characterization, for example Harris hip scores. In addition, the followup time of one year is very short for an endoprosthesis investigation, although this choice was in consideration of the limited life expectancy of these very elderly patients.

In view of the high incidence and the demographic development in the past few years, we have concentrated on these fractures.

\subsection{Conclusions}

Hemiprosthesis placement for displaced femoral neck fractures is a common and safe procedure. The goal of treatment is a quick operation and rapid mobilization. The cemented hemiprosthesis is implanted with a short operative time and allows immediate, full loading with a minimal dislocation rate. Despite recent decreases in hospital mortality, the risk of death remains more than twice as high within one year than that for uninjured patients of the same age.

\section{References}

1. Central bureau of Statistics Rheinland Pfalz . Abbreviated mortality table.;2012.

2. Stockle U, Lucke M, Haas NP. Certificate medical education: Femoral neck fracture. Dtsch Arztebl; 2005.

3. Bonnaire F, Lein T, Hohaus T, Weber A. [Prosthetic care of proximal femur fractures]. Unfallchirurg. 2005;108(5):387-99. doi:10.1007/s00113005-0942-z. [PubMed: 15864509] quiz 400.

4. Bonnaire F, Lein T, Engler KJ. [Treatment of femoral neck fractures]. Chirurg. 2008;79(6):595-611. doi: 10.1007/s00104-008-1551-5. [PubMed: 18523744] quiz 612.

5. Bonnaire F, Weber A. AWMF S2 Guideline: Femoral neck fractures in adult 2008. Available from: http://www.awmf.org/ uploads/tx_szleitlinien/012-0011_S2e_Schenkelhalsfraktur_des_ Erwachsenen.pdf.

6. Minne H, Pfeifer M, Wittenberg R. Femoral neck fractures in Germany: Prevention, Therapy, Incidence and socio-economic importance. Dtsch Artzebl; 2001.

7. Smektala R, Hahn S, Schrader P, Bonnaire F, Schulze Raestrup U, Siebert $\mathrm{H}$, et al. [Medial hip neck fracture: influence of pre-operative delay on the quality of outcome. Results of data from the external inhospital quality assurance within the framework of secondary data analysis]. Unfallchirurg. 2010;113(4):287-92. doi: 10.1007/s00113-0091674-2. [PubMed: 19756453]. 
8. Kirke PN, Sutton M, Burke H, Daly L. Outcome of hip fracture in older Irish women: a 2-year follow-up of subjects in a case-control study. Injury. 2002;33(5):387-91. [PubMed: 12095716].

9. Garden RS. Stability and union in subcapital fractures of the femur. Bone Joint J. 1964;46(4):630-47.

10. Pauwels F. Femoral neck fracture a mechanic problem. Stuttgart: Ferdinand Enke-Verlag; 1935.

11. Gjertsen JE, Engesaeter LB, Furnes O, Havelin LI, Steindal K, Vinje T, et al. The Norwegian Hip Fracture Register: experiences after the first 2 years and 15,576 reported operations. Acta Orthop. 2008;79(5):583-93. doi: 10.1080/17453670810016588. [PubMed: 18839363].

12. Gjertsen JE, Lie SA, Vinje T, Engesaeter LB, Hallan G, Matre K, et al. More re-operations after uncemented than cemented hemiarthroplasty used in the treatment of displaced fractures of the femoral neck: an observational study of 11,116 hemiarthroplasties from a national register. J Bone Joint Surg Br. 2012;94(8):1113-9. doi: 10.1302/0301620X.94B8.29155. [PubMed: 22844055].

13. Leonardsson O, Garellick G, Karrholm J, Akesson K, Rogmark C. Changes in implant choice and surgical technique for hemiarthroplasty. 21,346 procedures from the Swedish Hip Arthroplasty Register 2005-2009. Acta Orthop. 2012;83(1):7-13. doi: 10.3109/17453674.2011.641104. [PubMed: 22112151].

14. Seppanen M, Makela K, Virolainen P, Remes V, Pulkkinen P, Eskelinen A. Hip resurfacing arthroplasty: short-term survivorship of 4,401 hips from the Finnish Arthroplasty Register. Acta Orthop. 2012;83(3):207-13. doi: 10.3109/17453674.2012.693016. [PubMed: 22616745].

15. Prokop A, Chmielnicki M. Operation video. Technique of hemiprosthesis in femoral neck fracture 2015. Available from: https://www. youtube.com/watch? $\mathrm{v}=\mathrm{hseJCnPJ}-\mathrm{wI} \&$ feature=em-uploadowner.

16. Florschutz AV, Langford JR, Haidukewych GJ, Koval KJ. Femoral neck fractures: current management. J Orthop Trauma. 2015;29(3):121-9. doi: 10.1097/BOT.0000000000000291. [PubMed: 25635363].

17. Stoffel KK, Nivbrant B, Headford J, Nicholls RL, Yates PJ. Does a bipolar hemiprosthesis offer advantages for elderly patients with neck of femur fracture? A clinical trial with 261 patients. ANZ J Surg. 2013;83(4):249-54. doi: 10.1111/ans.12048. [PubMed: 23320780].

18. Schleicher I, Kordelle J, Jurgensen I, Haas H, Melzer C. [Femoral neck fractures in the elderly - bipolar hemiarthroplasty in total hip replacement]. Unfallchirurg. 2003;106(6):467-71. [PubMed: 14567174].

19. Muller CA, Bayer J, Szarzynski E, Sudkamp NP. [Implantation of bipolar prosthesis for treatment of medial femoral neck fractures in the elderly-clinical and radiographic outcome]. Zentralbl Chir 2008;133(6):590-6. doi: 10.1055/s-0028-1098711. [PubMed: 19090440].

20. Schliemann B, Seybold D, Gessmann J, Fehmer T, Schildhauer TA Muhr G. [Bipolar hemiarthroplasty in femoral neck fractures-impact of duration of surgery, time of day and the surgeon's experience on the complication rate]. Z Orthop Unfall. 2009;147(6):689-93. doi: 10.1055/s-0029-1186204. [PubMed: 19998222].

21. Schrader P, Rath T. [Volume-outcome-relationship in total hip replacement-literature review and model calculation of the health care situation]. Z Orthop Unfall. 2007;145(3):281-90. doi: 10.1055/s2007-965347. [PubMed: 17607624].

22. Schneppendahl J, Gehrmann SV, Hakimi M. Survival rate and clinical results after hemiarthroplasty in femoral neck fractures. Deutscher Kongress für Orthopädie und Unfallchirurgie 262010. Available from: http://www.egms.de/static/de/meetings/dkou2010/ 10dkou476.shtml.

23. Blomfeldt R, Tornkvist H, Eriksson K, Soderqvist A, Ponzer S, Tidermark J. A randomised controlled trial comparing bipolar hemiarthroplasty with total hip replacement for displaced intracapsular fractures of the femoral neck in elderly patients. J Bone Joint Surg Br. 2007;89(2):160-5. doi: 10.1302/0301-620X.89B2.18576. [PubMed: 17322427].

24. Avery PP, Baker RP, Walton MJ, Rooker JC, Squires B, Gargan MF, et al Total hip replacement and hemiarthroplasty in mobile, independent patients with a displaced intracapsular fracture of the femoral neck: a seven- to ten-year follow-up report of a prospective randomised controlled trial. J Bone Joint Surg Br. 2011;93(8):1045-8. doi: 10.1302/0301620X.93B8.27132. [PubMed: 21768626].

25. Zhao Y, Fu D, Chen K, Li G, Cai Z, Shi Y, et al. Outcome of hemiarthroplasty and total hip replacement for active elderly patients with displaced femoral neck fractures: a meta-analysis of 8 randomized clinical trials. PLoS One. 2014;9(5):e98071. doi: 10.1371/journal.pone.0098071. [PubMed: 24854195]. 